\title{
Anterior Spinal Artery Syndrome as Complication of Bronchial Artery Embolization
}

Ka Hong Chan MD, Chris White MD, FRCPC, and Jason K Wong MD, FRCPC

\begin{abstract}
About the Authors:
Ka Hong Chan is PGY-2 Internal Medicine, Department of Medicine, University of Calgary, Cumming School of Medicine, Calgary, Alberta. Chris White is Clinical Associate Professor, Department of Clinical Neurosciences, University of Calgary, Cumming School of Medicine, Calgary, Alberta. Jason Wong is Clinical Associate Professor, Cardiovascular and Interventional Radiology, University of Calgary, Cumming School of Medicine, Calgary, Alberta.

Correspondence to Chris White: Chris.White@albertahealthservices.ca

Submitted: December 11, 2017. Accepted: April 8, 2018. Published: November 9, 2018. DOI: 10.22374/cjgim.v13i4.260
\end{abstract}

\begin{abstract}
Bronchial artery embolization (BAE) has risen as one of the cornerstones of massive hemoptysis management. Though rare, spinal cord infarction is a potential complication. Here, we present a case of a 65-year-old gentleman who presented with acute weakness and was diagnosed with spinal cord infarction following BAE. This case will also review the pathophysiology of this adverse complication.
\end{abstract}

\section{RESUME}

L'embolisation de l'artère bronchique (BAE) est devenue l'un des piliers de la prise en charge massive de l'hémoptysie. Bien que rare, l'infarctus médullaire est une complication potentielle. Nous présentons ici le cas d'un homme âgé de 65 ans qui s'est présenté avec une faiblesse aiguë et un diagnostic d'infarctus médullaire à la suite d'une BAE. Ce cas examinera également la pathophysiologie de cette complication indésirable.

Traduit avec www.DeepL.com/Translator

Massive hemoptysis, usually defined as over $300 \mathrm{~mL}$ over 24 hours, is a potentially life-threatening condition that requires urgent medical attention. ${ }^{1}$ Traditionally, treatment was via either conservative or surgical management, but both carry mortality risks of up to $40 \%$ and $85 \%$ respectively. ${ }^{2,3}$ Bronchial artery embolization (BAE) has risen as one of the cornerstones of hemoptysis management, with long-term survival of $85 \% .{ }^{4}$ Though rare, spinal cord infarction is perhaps the most serious complication of BAE, with an incidence reported as high as $5 \%{ }^{5}$ Here, we present a rare case of anterior spinal artery syndrome following BAE.

A 65 year old gentleman with a history of Stage $3 \mathrm{~B}$ chronic kidney disease and coronary artery disease, on aspirin, presented with hemoptysis. He was known to have recurrent hemoptysis in the left upper lobe due to bronchiectasis from previous tuberculosis. He had previous BAE 3.5 years ago twice, once in the left upper lobe bronchial artery and another in the first and second intercostal arteries. Most recently, he presented with recurrent hemoptysis and unfortunately failed medical management with broad spectrum antibiotics and transxenamic acid that had helped him previously. Hence, he underwent another embolization.

Via a right femoral approach, the left BA was identified. Because of shunting to the pulmonary artery, a small amount of larger 700-900 $\mu \mathrm{m}$ microsphere particles were first administered before embolization was completed with $300-500 \mu \mathrm{m}$ particles. A similar technique was done on the milder right side. No spinal arteries were identified throughout. A re-look of a different left 
sided BA conducted subsequently continued to demonstrate abnormal neovascularity. Further embolization was conducted by inserting a microcatheter past some tiny branch origins, which was suspected to provide supply to the spinal cord (Figure 1).

The next day, the patient complained of left sided weakness and numbness on the belly. Strength examination demonstrated $4 / 5$ weakness on the left lower as well as decreased pain and temperature sensation up to the T5-T6 area on the right. The rest of his physical examination, including reflexes and tone, were normal. Magnetic resonance imaging of his thoracic spine demonstrated a subtle linear T2/STIR hyperintensity within the anterior spinal cord at the T6-T8 level consistent with cord ischemia (Figure 2). Fortunately, his functional deficits were mild, and he made a good recovery with rehabilitative therapy.
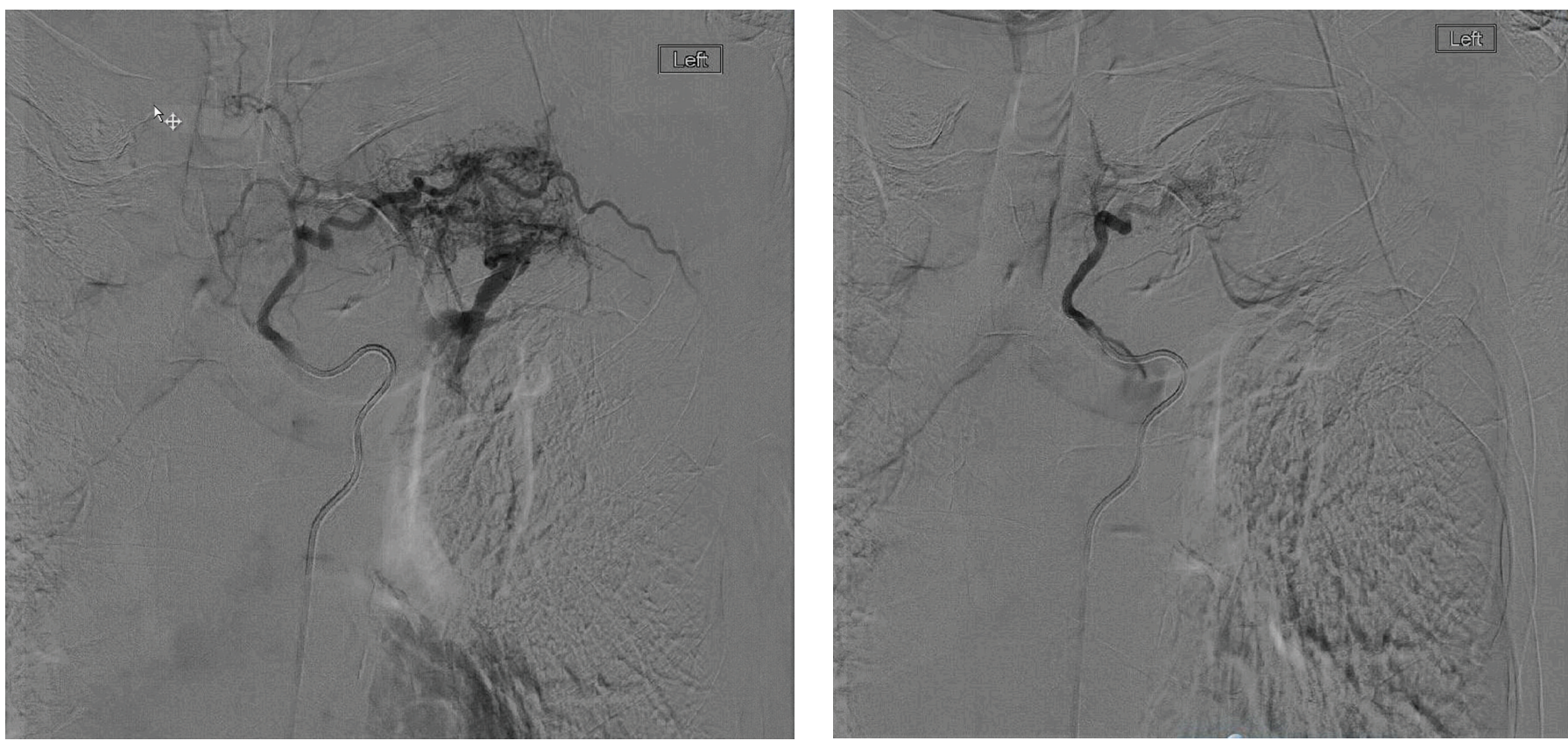

Figure 1. Left bronchial artery angiogram demonstrating neovascularity with shunting to the pulmonary arteries with possible tiny spinal artery branches (left) before and (right) after embolization.
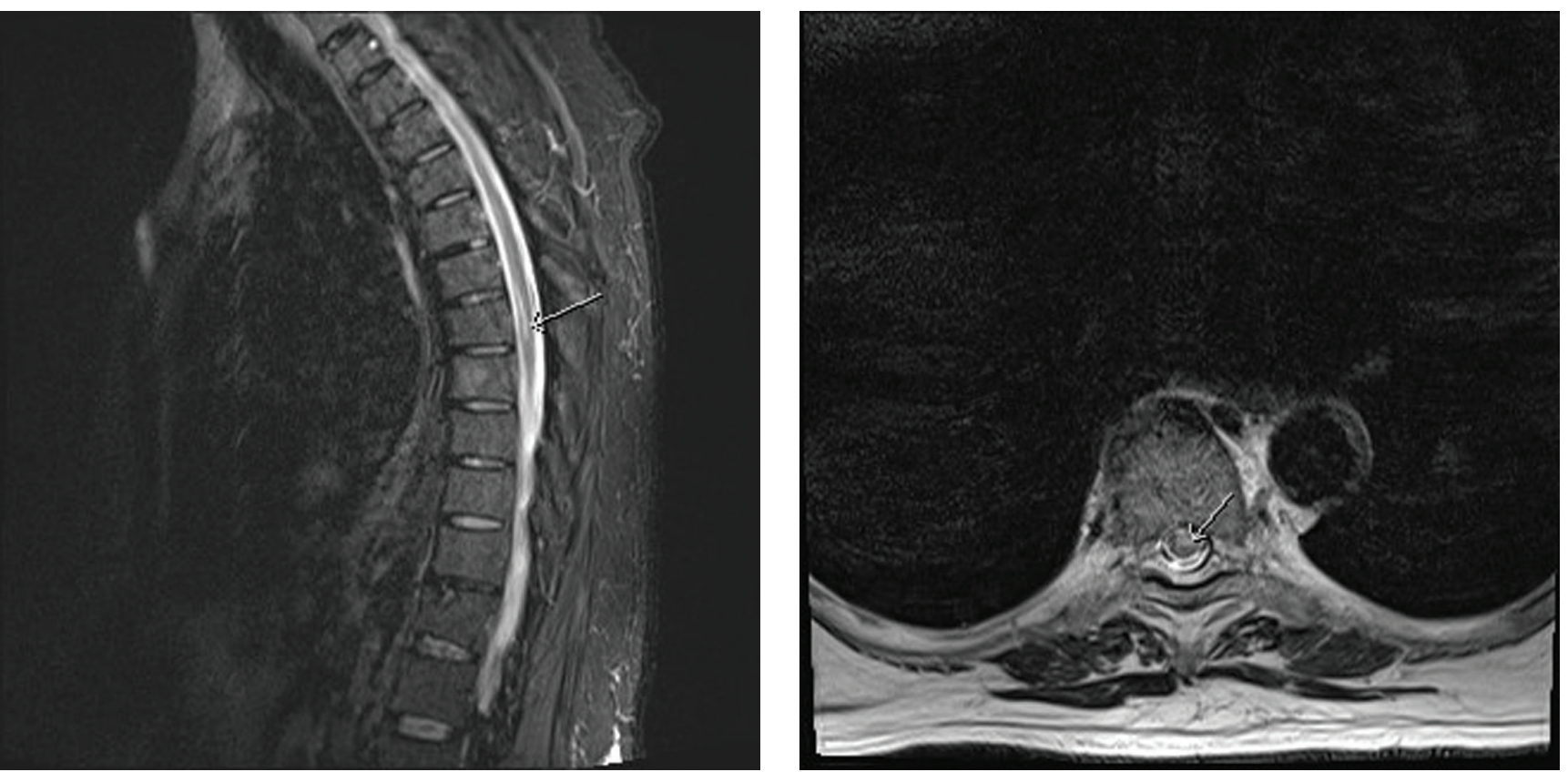

Figure 2. Saggital linear T2/STIR hyperdensity within the anterior spinal cord at approximately T7 and corresponding T2 signal increase on axial image representing cord ischemia. 


\section{Discussion}

BAE has now become the mainline treatment for massive hemoptysis as many of these patients are poor surgical candidates due to their underlying chronic pulmonary disease (e.g., interstitial lung disease, cystic fibrosis, chronic infections such as tuberculosis). Identification of the $\mathrm{BA}$ is the cornerstone of this treatment. However, there is significant anatomical variability in the branching pattern and multiple possible anomalous origins of the BA. ${ }^{1,3,6}$ The diffuse involvement of these patients' underlying pulmonary disease make the exact site of hemorrhage is difficult to confidently isolate. Therefore, because of the life-threatening nature of massive hemoptysis, multiple BA are frequently treated to reduce bleeding risk.

Radicular and medullary arteries, which reinforce blood flow to the anterior spinal artery, can arise from the BA as well as the intercostobronchial trunk and intercostal artery. Hence, a side effect when embolizing the BA can be spinal cord infarction. The latter is especially important, and hence, should be identified during angiography by looking for the characteristic "hairpin" configuration. ${ }^{1,6}$ In reality, spinal arteries are difficult to observe and increased risk of ischemia can occur with repeated embolization as with our case. Moreover, care must also be taken to ensure that the artery of Adamkiewicz is not found the in upper thorax prior to embolization. Although it is mostly found T8 or lower, it can be found as high as T5. ${ }^{7}$

Great care and multiple angiographies were conducted in our case to visualize and avoid the spinal arteries. Despite this, our patient still suffered from anterior spinal artery syndrome post BAE. In reality, spinal arteries are actually rarely observed and increased risk of ischemia can occur with repeated embolization as with our case. ${ }^{6}$
In conclusion, although BAE has now arisen as a relatively safe procedure with good long-term outcomes, spinal cord ischemia can be a rare but serious complication in which patients should be counselled on prior to the procedure.

\section{Author Contributions}

Ka Hong Chan: design of case report, drafting and revising the manuscript. Jason K. Wong and Chris White design of case report and revising manuscript.

\section{Study Funding}

No targeted funding reported.

\section{Disclosures}

Ka Hong Chan and Chris White do not have anything to disclose. Jason K. Wong receives proctoring honoraria from Cook Medical and is a paid speaker by Cook Medical and Boston Scientific.

\section{References}

1. Marshall TJ and Jackson JE. Vascular intervention in the thorax: bronchial artery embolization for hemoptysis. Eur Radiol 1997;7(8):1221-7.

2. Burke CT and Mauro MA. Bronchial artery embolization. Semin Intervent Radiol 2004;21(1):43-8.

3. Sopko DR and Smith TP. Bronchial artery embolization for hemoptysis. Semin Intervent Radiol 2011;28(1):48-62.

4. Pathak V, Stavas JM, Ford HJ, et al. Long-term outcomes of the bronchial artery embolization are diagnosis dependent. Lung India 2016;33(1):3-8.

5. Lorenz J, Sheth D, and Patel J. Bronchial artery embolization. Semin Intervent Radiol 2012;29(3):155-60.

6. Yoon W, Kim JK, Kim YH, et al. Bronchial and nonbronchial systemic artery embolization for life-threatening hemoptysis: a comprehensive review. Radiographics 2002;22(6):1395-409.

7. Stoll JF and Bettmann MA. Bronchial artery embolization to control hemoptysis: a review. Cardiovasc Intervent Radiol 1988;11(5):263-9. 